\title{
A half-discrete Hilbert-type inequality with the non-monotone kernel and the best constant factor
}

Dongmei Xin ${ }^{*}$ and Bicheng Yang

\section{"Correspondence:}

xdm77108@gdei.edu.cn

Department of Mathematics,

Guangdong Education University,

Guangzhou, Guangdong 510303,

People's Republic of China

\begin{abstract}
By introducing two pairs of conjugate exponents and using the improved Euler-Maclaurin summation formula, we estimate the weight functions and obtain a half-discrete Hilbert-type inequality with the non-monotone kernel and the best constant factor. We also consider its equivalent forms.
\end{abstract}

MSC: $26 \mathrm{D} 15$

Keywords: Hilbert-type inequality; conjugate exponent; Hölder's inequality; best constant factor; equivalent form

\section{Introduction}

If $a_{n}, b_{n} \geq 0$, such that $0<\sum_{n=1}^{\infty} a_{n}^{2}<\infty$ and $0<\sum_{n=1}^{\infty} b_{n}^{2}<\infty$, then we have the famous Hilbert's inequality as follows (cf. [1]):

$$
\sum_{n=1}^{\infty} \sum_{m=1}^{\infty} \frac{a_{m} b_{n}}{m+n}<\pi\left(\sum_{n=1}^{\infty} a_{n}^{2} \sum_{n=1}^{\infty} b_{n}^{2}\right)^{\frac{1}{2}}
$$

where the constant factor $\pi$ is the best possible.

Under the same condition of (1), Xin et al. [2] gave the following inequality:

$$
\sum_{n=1}^{\infty} \sum_{m=1}^{\infty} \frac{|\ln (m / n)|}{m+n} a_{m} b_{n}<c_{0}\left(\sum_{n=1}^{\infty} a_{n}^{2} \sum_{n=1}^{\infty} b_{n}^{2}\right)^{\frac{1}{2}}
$$

where the constant factor $c_{0}=8 \sum_{n=1}^{\infty} \frac{(-1)^{n-1}}{(2 n-1)^{2}}=7.3277^{+}$is the best possible. And Yang [3] gave the integral analogues of (2).

In 1934, Hardy et al. [1] established a few results on the half-discrete Hilbert-type inequalities with the non-homogeneous kernel (see Theorem 351). But they did not prove that the constant factors are the best possible. However, Yang [4] gave a result by introducing an interval variable and proved that the constant factor is the best possible. Recently, Yang et al. [5-9] gave some half-discrete Hilbert-type inequalities and their reverses with the monotone kernels and best constant factors.

(0) 2012 Xin and Yang; licensee Springer. This is an Open Access article distributed under the terms of the Creative Commons Attribution License (http://creativecommons.org/licenses/by/2.0), which permits unrestricted use, distribution, and reproduction in any medium, provided the original work is properly cited. 
Recently, Yang [10] gave the following half-discrete Hilbert-type inequality with the nonmonotone kernel and the best constant factor 8:

$$
\sum_{n=1}^{\infty} \int_{1}^{\infty} \frac{|\ln (x / n)| a_{n}}{\max \{x, n\}} f(x) d x<8\left(\sum_{n=1}^{\infty} a_{n}^{2} \int_{1}^{\infty} f^{2}(x) d x\right)^{\frac{1}{2}} .
$$

Obviously, for a half-discrete Hilbert-type inequality with the monotone kernel, it is easy to build the relating inequality by estimating the series form and the integral form of weight functions. However, for a half-discrete Hilbert-type inequality with the non-monotone kernel, it is much more difficult to prove.

In this paper, by using the way of weight functions, we give a new half-discrete Hilberttype inequality with the non-monotone kernel as follows:

$$
\sum_{n=1}^{\infty} a_{n} \int_{1}^{\infty} \frac{\left|\ln \left(\frac{x}{n}\right)\right| f(x)}{x+n} d x<8 \sum_{k=0}^{\infty} \frac{(-1)^{k}}{(2 k+1)^{2}}\left(\sum_{n=1}^{\infty} a_{n}^{2} \int_{1}^{\infty} f^{2}(x)\right)^{\frac{1}{2}},
$$

where the constant factor $8 \sum_{k=0}^{\infty} \frac{(-1)^{k}}{(2 k+1)^{2}}$ is the best possible. The main objective of this paper is to build the best extension of (4) with parameters and equivalent forms.

\section{Some lemmas}

Lemma 2.1 If $x_{1} \in \mathbf{R}, n_{1} \in \mathbf{Z}$ (Z is the set of non-negative integers), $\left[x_{1}\right]=n_{1}, \rho(y)=y-[y]-\frac{1}{2}$ $(y \in \mathbf{R})$ is the Bernoulli function of first order [11], then we have (cf. [10])

$$
\int_{n_{1}}^{x_{1}} \rho(y) d y=-\frac{\varepsilon_{1}}{8} \quad\left(\varepsilon_{1} \in[0,1]\right) .
$$

Lemma 2.2 If $r>1, \frac{1}{r}+\frac{1}{s}=1, f(x, y):=\frac{\left|\ln \left(\frac{x}{y}\right)\right|}{x+y}\left(\frac{x}{y}\right)^{\frac{1}{r}}(x, y \in(0, \infty)), \mathbf{N}$ is the set of positive integers, define the weight functions as follows:

$$
\begin{aligned}
& \omega(n):=\int_{1}^{\infty} \frac{\left|\ln \left(\frac{x}{n}\right)\right|}{x+n}\left(\frac{n}{x}\right)^{\frac{1}{s}} d x \quad(n \in \mathbf{N}), \\
& \varpi(x):=\sum_{n=1}^{\infty} \frac{\left|\ln \left(\frac{x}{n}\right)\right|}{x+n}\left(\frac{x}{n}\right)^{\frac{1}{r}} \quad(x \in[1, \infty)) .
\end{aligned}
$$

Then we have

$$
\omega(n)<c_{r}:=\sum_{k=0}^{\infty}(-1)^{k}\left[\frac{1}{\left(k+\frac{1}{r}\right)^{2}}+\frac{1}{\left(k+\frac{1}{s}\right)^{2}}\right], \quad \varpi(x)<c_{r} .
$$

Proof Setting $u=\frac{x}{n}$ in (6), we have

$$
\omega(n)=\int_{\frac{1}{n}}^{\infty} \frac{|\ln u|}{u+1} u^{-\frac{1}{s}} d u<\int_{0}^{\infty} \frac{|\ln u|}{u+1} u^{-\frac{1}{s}} d u=c_{r} .
$$

Setting $u=\frac{y}{x}$, then it follows

$$
\int_{0}^{1} f(x, y) d y=\int_{0}^{\frac{1}{x}} \frac{(-\ln u)}{u+1} u^{-\frac{1}{r}} d u>s \int_{0}^{\frac{1}{x}} \frac{(-\ln u)}{\frac{1}{x}+1} d u^{-\frac{1}{r}+1}=\frac{x^{\frac{1}{r}}}{x+1}\left(s \ln x+s^{2}\right) .
$$


For $1 \leq y<x, f(x, y)=-\frac{\ln \left(\frac{y}{x}\right)}{x+y}\left(\frac{x}{y}\right)^{\frac{1}{r}}$, we have

$$
\begin{aligned}
f_{y}^{\prime}(x, y)= & x^{\frac{1}{r}}\left[-\frac{1}{(y+x) y^{1+\frac{1}{r}}}+\frac{\ln \left(\frac{y}{x}\right)}{(y+x)^{2} y^{\frac{1}{r}}}+\frac{\ln \left(\frac{y}{x}\right)}{r(y+x) y^{1+\frac{1}{r}}}\right] \\
= & -x^{\frac{1}{r}}\left[\frac{1}{(y+x) y^{1+\frac{1}{r}}}+\frac{2 x \ln \left(\frac{y}{x}\right)}{(y-x)(y+x)^{2} y^{\frac{1}{r}}}+\frac{2 x \ln \left(\frac{y}{x}\right)}{r(y-x)(y+x) y^{1+\frac{1}{r}}}\right] \\
& +x^{\frac{1}{r}}\left[\frac{\ln \left(\frac{y}{x}\right)}{(y-x)(y+x) y^{\frac{1}{r}}}+\frac{\ln \left(\frac{y}{x}\right)}{r(y-x) y^{1+\frac{1}{r}}}\right] .
\end{aligned}
$$

For $y \geq x, f(x, y)=\frac{\ln \left(\frac{y}{x}\right)}{x+y}\left(\frac{x}{y}\right)^{\frac{1}{r}}$, we find

$$
\begin{aligned}
f_{y}^{\prime}(x, y)= & x^{\frac{1}{r}}\left[\frac{1}{(y+x) y^{1+\frac{1}{r}}}-\frac{\ln \left(\frac{y}{x}\right)}{(y+x)^{2} y^{\frac{1}{r}}}-\frac{\ln \left(\frac{y}{x}\right)}{r(y+x) y^{1+\frac{1}{r}}}\right] \\
= & x^{\frac{1}{r}}\left[\frac{1}{(y+x) y^{1+\frac{1}{r}}}+\frac{2 x \ln \left(\frac{y}{x}\right)}{(y-x)(y+x)^{2} y^{\frac{1}{r}}}+\frac{2 x \ln \left(\frac{y}{x}\right)}{r(y-x)(y+x) y^{1+\frac{1}{r}}}\right] \\
& -x^{\frac{1}{r}}\left[\frac{\ln \left(\frac{y}{x}\right)}{(y-x)(y+x) y^{\frac{1}{r}}}+\frac{\ln \left(\frac{y}{x}\right)}{r(y-x) y^{1+\frac{1}{r}}}\right] .
\end{aligned}
$$

Define two functions as follows:

$$
\begin{aligned}
& g(y)= \begin{cases}g_{1}(y)=x^{\frac{1}{r}}\left[\frac{1}{(y+x) y^{1+\frac{1}{r}}}+\frac{2 x \ln \left(\frac{y}{x}\right)}{(y-x)(y+x)^{2} y^{\frac{1}{r}}}+\frac{2 x \ln \left(\frac{y}{x}\right)}{r(y-x)(y+x) y^{1+\frac{1}{r}}}\right], & y<x ; \\
g_{2}(y)=x^{\frac{1}{r}}\left[\frac{\ln \left(\frac{y}{x}\right)}{(y-x)(y+x) y^{\frac{1}{r}}}+\frac{\ln \left(\frac{y}{x}\right)}{r(y-x) y^{1+\frac{1}{r}}}\right], & y \geq x,\end{cases} \\
& h(y)= \begin{cases}h_{1}(y)=x^{\frac{1}{r}}\left[\frac{\ln \left(\frac{y}{x}\right)}{(y-x)(y+x) y^{\frac{1}{r}}}+\frac{\ln \left(\frac{y}{x}\right)}{r(y-x) y^{1+\frac{1}{r}}}\right], & y<x ; \\
h_{2}(y)=x^{\frac{1}{r}}\left[\frac{1}{(y+x) y^{1+\frac{1}{r}}}+\frac{2 x \ln \left(\frac{y}{x}\right)}{(y-x)(y+x)^{2} y^{\frac{1}{r}}}+\frac{2 x \ln \left(\frac{y}{x}\right)}{r(y-x)(y+x) y^{1+\frac{1}{r}}}\right], & y \geq x .\end{cases}
\end{aligned}
$$

Then we have $-f_{y}^{\prime}(x, y)=g(y)-h(y)$. Setting $a=\frac{1}{2 x^{2}}, b=-\frac{1}{2 x^{2}}$, then $a-b=\frac{1}{x^{2}}$. Define two functions as follows:

$$
\tilde{g}(y)=\left\{\begin{array}{ll}
g_{1}(y)-a, & y<x ; \\
g_{2}(y), & y \geq x,
\end{array} \quad \tilde{h}(y)= \begin{cases}h_{1}(y)-b, & y<x \\
h_{2}(y), & y \geq x\end{cases}\right.
$$

Since $g_{1}(x-0)-a=g_{2}(x), h_{1}(x-0)-b=h_{2}(x)$, then both $\tilde{g}(y)$ and $\tilde{h}(y)(y \in[1, \infty))$ are decreasing and continuous. Besides $y=x$, we have $(-1)^{i} \tilde{g}^{(i)}(y) \geq 0,(-1)^{i} \tilde{h}^{(i)}(y) \geq 0(i=0,1)$, and $\tilde{g}(\infty)=\tilde{h}(\infty)=0$. By the improved Euler-Maclaurin summation formula (cf. [11], Theorem 2.2.2) and (5), for $\varepsilon_{1} \in[0,1], \varepsilon_{i} \in(0,1)(i=2,3)$, it follows

$$
\begin{aligned}
& -\int_{1}^{\infty} \rho(y) f_{y}^{\prime}(x, y) d y \\
& \quad=\int_{1}^{\infty} \rho(y) g(y) d y-\int_{1}^{\infty} \rho(y) h(y) d y \\
& \quad=\int_{1}^{\infty} \rho(y) \tilde{g}(y) d y+a \int_{1}^{x} \rho(y) d y-\left[\int_{1}^{\infty} \rho(y) \tilde{h}(y) d y+b \int_{1}^{x} \rho(y) d y\right]
\end{aligned}
$$




$$
\begin{aligned}
& =\int_{1}^{\infty} \rho(y) \tilde{g}(y) d y-\int_{1}^{\infty} \rho(y) \tilde{h}(y) d y+(a-b)\left[\int_{1}^{[x]} \rho(y) d y+\int_{[x]}^{x} \rho(y) d y\right] \\
& =-\frac{\varepsilon_{2}}{8} \tilde{g}(1)+\frac{\varepsilon_{3}}{8} \tilde{h}(1)-\frac{\varepsilon_{1}}{8}(a-b)=-\frac{\varepsilon_{2}}{8}\left(g_{1}(1)-a\right)+\frac{\varepsilon_{3}}{8}\left(h_{1}(1)-b\right)-\frac{\varepsilon_{1}}{8}(a-b) .
\end{aligned}
$$

Since $g_{1}(1)-a \geq g_{1}(x-0)-a=g_{2}(x)>0, h_{1}(1)-b \geq h_{1}(x-0)-b=h_{2}(x)>0$, then we have

$$
\begin{aligned}
-\int_{1}^{\infty} \rho(y) f_{y}^{\prime}(x, y) d y & >-\frac{1}{8}\left(g_{1}(1)-a\right)-\frac{1}{8}(a-b) \\
& =-\frac{x^{\frac{1}{r}}}{8(x+1)}-\frac{x^{1+\frac{1}{r}} \ln x}{4(x+1)^{2}(x-1)}-\frac{x^{1+\frac{1}{r}} \ln x}{4 r(x+1)(x-1)}-\frac{1}{16 x^{2}}
\end{aligned}
$$

By the improved Euler-Maclaurin summation formula [11], we have

$$
\begin{aligned}
\varpi(x) & =\sum_{k=1}^{\infty} f(x, k)=\int_{1}^{\infty} f(x, y) d y+\frac{1}{2} f(x, 1)+\int_{1}^{\infty} \rho(y) f_{y}^{\prime}(x, y) d y \\
& =\int_{0}^{\infty} f(x, y) d y-\left(\int_{0}^{1} f(x, y) d y-\frac{1}{2} f(x, 1)-\int_{1}^{\infty} \rho(y) f_{y}^{\prime}(x, y) d y\right)=c_{r}-\theta(x),
\end{aligned}
$$

where

$$
\theta(x):=\int_{0}^{1} f(x, y) d y-\frac{1}{2} f(x, 1)-\int_{1}^{\infty} \rho(y) f_{y}^{\prime}(x, y) d y
$$

Since $-\frac{1}{2} f(x, 1)=-\frac{x^{\frac{1}{r}} \ln x}{2(x+1)}$, in view of (9), (10), (i) for $1 \leq x<2,-\frac{\ln x}{x-1} \geq-1$, we have

$$
\begin{aligned}
\theta(x)> & \frac{x^{\frac{1}{r}}}{x+1}\left(s \ln x+s^{2}\right)-\frac{x^{\frac{1}{r}} \ln x}{2(x+1)}-\frac{x^{\frac{1}{r}}}{8(x+1)} \\
& -\frac{x^{1+\frac{1}{r}} \ln x}{4(x+1)^{2}(x-1)}-\frac{x^{1+\frac{1}{r}} \ln x}{4 r(x+1)(x-1)}-\frac{1}{16 x^{2}} \\
\geq & \frac{x^{\frac{1}{r}} \ln x}{(x+1)}\left(s-\frac{1}{2}\right)+\frac{x^{\frac{1}{r}}}{x+1}\left[s^{2}-\frac{1}{8}-\frac{x}{4(x+1)}-\frac{x}{4 r}-\frac{x+1}{16 x^{2+\frac{1}{r}}}\right] \\
> & \frac{x^{\frac{1}{r}}}{(x+1)}\left(1-\frac{1}{8}-\frac{1}{4}-\frac{1}{2}-\frac{1}{8}\right)=0 ;
\end{aligned}
$$

(ii) for $x \geq 2,-\frac{1}{x-1} \geq-\frac{2}{x}$, we have

$$
\begin{aligned}
\theta(x) & \geq \frac{x^{\frac{1}{r}} \ln x}{(x+1)}\left[s-\frac{1}{2}-\frac{1}{2(x+1)}-\frac{1}{2 r}\right]+\frac{x^{\frac{1}{r}}}{x+1}\left(s^{2}-\frac{1}{8}-\frac{x+1}{16 x^{2+\frac{1}{r}}}\right) \\
& >\frac{x^{\frac{1}{r}} \ln x}{(x+1)}\left(s-\frac{1}{2}-\frac{1}{6}-\frac{1}{2 r}\right)=\frac{(6 s+2 r-3) x^{\frac{1}{r}} \ln x}{6 r(x+1)}>0 .
\end{aligned}
$$

Hence, for $x \geq 1$, we have $\theta(x)>0$, it follows $\varpi(x)<c_{r}$. The lemma is proved. 
Lemma 2.3 As the assumption of Lemma 2.2, if $0<\varepsilon<\frac{p}{r}, R=\left(\frac{1}{r}-\frac{\varepsilon}{p}\right)^{-1}, S=\left(\frac{1}{s}+\frac{\varepsilon}{p}\right)^{-1}$, then we have

$$
\bar{I}:=\sum_{n=1}^{\infty} n^{\frac{1}{S}-\varepsilon-1} \int_{1}^{\infty} \frac{\left|\ln \left(\frac{x}{n}\right)\right|}{x+n} x^{\frac{1}{R}-1} d x \geq \frac{c_{R}}{\varepsilon}-O(1)
$$

Proof It is obvious that $R>1, \frac{1}{R}+\frac{1}{S}=1$. Setting $u=\frac{x}{n}$, we have

$$
\begin{aligned}
\bar{I} & =\sum_{n=1}^{\infty} n^{-\varepsilon-1} \int_{\frac{1}{n}}^{\infty} \frac{|\ln u|}{u+1} u^{\frac{1}{R}-1} d u \\
& =\sum_{n=1}^{\infty} n^{-\varepsilon-1}\left[\int_{0}^{\infty} \frac{|\ln u|}{u+1} u^{\frac{1}{R}-1} d u+\int_{0}^{\frac{1}{n}} \frac{\ln u}{u+1} u^{\frac{1}{R^{-}}-1} d u\right] \\
& >c_{R} \int_{1}^{\infty} x^{-\varepsilon-1} d x+R \sum_{n=1}^{\infty} n^{-\varepsilon-1} \int_{0}^{\frac{1}{n}} \ln u d u^{\frac{1}{R}} \\
& =\frac{c_{R}}{\varepsilon}-\left[R \sum_{n=1}^{\infty} \frac{\ln n}{n^{1+\varepsilon+\frac{1}{R}}}+R^{2} \sum_{n=1}^{\infty} \frac{1}{n^{1+\varepsilon+\frac{1}{R}}}\right] \\
& =\frac{c_{R}}{\varepsilon}-O(1) \quad\left(\varepsilon \rightarrow 0^{+}\right) .
\end{aligned}
$$

The lemma is proved.

Lemma 2.4 If $p, r>1, \frac{1}{r}+\frac{1}{s}=\frac{1}{p}+\frac{1}{q}=1, a_{n} \geq 0, f(x)$ is a non-negative measurable function, then we have

$$
\begin{aligned}
& J_{1}:=\sum_{n=1}^{\infty} n^{\frac{p}{s}-1}\left[\int_{1}^{\infty} \frac{\left|\ln \left(\frac{x}{n}\right)\right| f(x)}{x+n} d x\right]^{p} \leq c_{r}^{p} \int_{1}^{\infty} x^{\frac{p}{s}-1} f^{p}(x) d x, \\
& J_{2}:=\int_{1}^{\infty} x^{\frac{q}{r}-1}\left[\sum_{n=1}^{\infty} \frac{\left|\ln \left(\frac{x}{n}\right)\right| a_{n}}{x+n}\right]^{q} d x \leq c_{r}^{q} \sum_{n=1}^{\infty} n^{\frac{q}{r}-1} a_{n}^{q} .
\end{aligned}
$$

Proof By Hölder's inequality [12], in view of (6) and (7), we have

$$
\begin{aligned}
& {\left[\int_{1}^{\infty} \frac{\left|\ln \left(\frac{x}{n}\right)\right| f(x)}{x+n} d x\right]^{p}} \\
& \quad=\left\{\int_{1}^{\infty} \frac{\left|\ln \left(\frac{x}{n}\right)\right|}{x+n}\left[\frac{x^{\frac{1}{s q}}}{n^{\frac{1}{r p}}} f(x)\right]\left[\frac{n^{\frac{1}{r p}}}{x^{\frac{1}{s q}}}\right] d x\right\}^{p} \\
& \quad \leq \int_{1}^{\infty} \frac{\left|\ln \left(\frac{x}{n}\right)\right|}{x+n}\left(\frac{x}{n}\right)^{\frac{1}{r}} x^{\frac{p}{s}-1} f^{p}(x) d x\left[\int_{1}^{\infty} \frac{\left|\ln \left(\frac{x}{n}\right)\right|}{x+n}\left(\frac{n}{x}\right)^{\frac{1}{s}} n^{\frac{q}{r}-1} d x\right]^{p-1} \\
& \quad=\int_{1}^{\infty} \frac{\left|\ln \left(\frac{x}{n}\right)\right|}{x+n}\left(\frac{x}{n}\right)^{\frac{1}{r}} x^{\frac{p}{s}-1} f^{p}(x) d x\left[n^{\frac{q}{r}-1} \omega(n)\right]^{p-1} \\
& \quad \leq n^{-\frac{p}{s}+1} c_{r}^{p-1} \int_{1}^{\infty} \frac{\left|\ln \left(\frac{x}{n}\right)\right|}{x+n}\left(\frac{x}{n}\right)^{\frac{1}{r}} x^{\frac{p}{s}-1} f^{p}(x) d x, \\
& J_{1} \leq c_{r}^{p-1} \sum_{n=1}^{\infty} \int_{1}^{\infty} \frac{\left|\ln \left(\frac{x}{n}\right)\right|}{x+n}\left(\frac{x}{n}\right)^{\frac{1}{r}} x^{\frac{p}{s}-1} f^{p}(x) d x
\end{aligned}
$$




$$
\begin{aligned}
& =c_{r}^{p-1} \int_{1}^{\infty}\left[\sum_{n=1}^{\infty} \frac{\left|\ln \left(\frac{x}{n}\right)\right|}{x+n}\left(\frac{x}{n}\right)^{\frac{1}{r}}\right] x^{\frac{p}{s}-1} f^{p}(x) d x \\
& =c_{r}^{p-1} \int_{1}^{\infty} \varpi(x) x^{\frac{p}{s}-1} f^{p}(x) d x \leq c_{r}^{p} \int_{1}^{\infty} x^{\frac{p}{s}-1} f^{p}(x) d x .
\end{aligned}
$$

Hence we have (12). Still by Hölder's inequality [12], (6) and (7), we have

$$
\begin{aligned}
& {\left[\sum_{n=1}^{\infty} \frac{\left|\ln \left(\frac{x}{n}\right)\right| a_{n}}{x+n}\right]^{q}=\left\{\sum_{n=1}^{\infty} \frac{\left|\ln \left(\frac{x}{n}\right)\right|}{x+n}\left[\frac{x^{\frac{1}{s q}}}{n^{\frac{1}{r p}}}\right]\left[\frac{n^{\frac{1}{r p}}}{x^{\frac{1}{s q}}} a_{n}\right]\right\}^{q}} \\
& \leq\left[\varpi(x) \sum_{n=1}^{\infty} x^{\frac{p}{s}-1}\right]^{q-1} \sum_{n=1}^{\infty} \frac{\left|\ln \left(\frac{x}{n}\right)\right|}{x+n}\left(\frac{x}{n}\right)^{\frac{1}{r}} n^{\frac{q}{r}-1} a_{n}^{q} \\
& \leq x^{-\frac{q}{r}+1} c_{r}^{q-1} \sum_{n=1}^{\infty} \frac{\left|\ln \left(\frac{x}{n}\right)\right|}{x+n}\left(\frac{x}{n}\right)^{\frac{1}{r}} n^{\frac{q}{r}-1} a_{n}^{q}, \\
& J_{2} \leq c_{r}^{q-1} \int_{1}^{\infty}\left[\sum_{n=1}^{\infty} \frac{\left|\ln \left(\frac{x}{n}\right)\right|}{x+n}\left(\frac{x}{n}\right)^{\frac{1}{r}} n^{\frac{q}{r}-1} a_{n}^{q}\right] d x \\
& =c_{r}^{q-1} \sum_{n=1}^{\infty}\left[\int_{1}^{\infty} \frac{\left|\ln \left(\frac{x}{n}\right)\right|}{x+n}\left(\frac{x}{n}\right)^{\frac{1}{r}} d x\right] n^{\frac{q}{r}-1} a_{n}^{q} \\
& =c_{r}^{q-1} \sum_{n=1}^{\infty} \omega(n) n^{\frac{q}{r}-1} a_{n}^{q} \leq c_{r}^{q} \sum_{n=1}^{\infty} n^{\frac{q}{r}-1} a_{n}^{q} .
\end{aligned}
$$

Then we have (13). The lemma is proved.

\section{Main results and applications}

Theorem 3.1 If $p, r>1, \frac{1}{p}+\frac{1}{q}=\frac{1}{r}+\frac{1}{s}=1, a_{n}, f(x) \geq 0$ such that $0<\int_{1}^{\infty} x^{\frac{p}{s}-1} f^{p}(x) d x<\infty$, $\sum_{n=1}^{\infty} n^{\frac{q}{r}-1} a_{n}^{q}<\infty$, then we have the following equivalent inequalities:

$$
\begin{aligned}
I & :=\sum_{n=1}^{\infty} a_{n} \int_{1}^{\infty} \frac{\left|\ln \left(\frac{x}{n}\right)\right| f(x)}{x+n} d x=\int_{1}^{\infty} f(x) \sum_{n=1}^{\infty} \frac{\left|\ln \left(\frac{x}{n}\right)\right| a_{n}}{x+n} d x \\
& <c_{r}\left\{\int_{1}^{\infty} x^{\frac{p}{s}-1} f^{p}(x) d x\right\}^{\frac{1}{p}}\left\{\sum_{n=1}^{\infty} n^{\frac{q}{r}-1} a_{n}^{q}\right\}^{\frac{1}{q}}, \\
J_{1} & =\sum_{n=1}^{\infty} n^{\frac{p}{s}-1}\left[\int_{1}^{\infty} \frac{\left|\ln \left(\frac{x}{n}\right)\right| f(x)}{x+n} d x\right]^{p}<c_{r}^{p} \int_{1}^{\infty} x^{\frac{p}{s}-1} f^{p}(x) d x, \\
J_{2} & =\int_{1}^{\infty} x^{\frac{q}{r}-1}\left[\sum_{n=1}^{\infty} \frac{\left|\ln \left(\frac{x}{n}\right)\right| a_{n}}{x+n}\right]^{q} d x<c_{r}^{q} \sum_{n=1}^{\infty} n^{\frac{q}{r}-1} a_{n}^{q},
\end{aligned}
$$

where the constant factors $c_{r}=\sum_{k=0}^{\infty}(-1)^{k}\left[\frac{1}{\left(k+\frac{1}{r}\right)^{2}}+\frac{1}{\left(k+\frac{1}{s}\right)^{2}}\right], c_{r}^{p}$, $c_{r}^{q}$ are the best possible.

Proof By the Lebesgue term-by-term integration theorem [13], there are two kinds of representation in (14). By the conditions of Theorem 3.1, (12) takes the form of a strict inequal- 
ity, and we have (15). By Hölder's inequality [12], we have

$$
I=\sum_{n=1}^{\infty}\left[n^{\frac{1}{s}-\frac{1}{p}} \int_{1}^{\infty} \frac{\left|\ln \left(\frac{x}{n}\right)\right| f(x)}{x+n} d x\right]\left[n^{-\frac{1}{s}+\frac{1}{p}} a_{n}\right] \leq J_{1}^{\frac{1}{p}}\left\{\sum_{n=1}^{\infty} n^{\frac{q}{r}-1} a_{n}^{q}\right\}^{\frac{1}{q}}
$$

By (15), we have (14). On the other hand, suppose that (14) is valid. Setting $a_{n}:=$ $n^{\frac{p}{s}-1}\left[\int_{1}^{\infty} \frac{\left|\ln \left(\frac{x}{n}\right)\right| f(x)}{x+n} d x\right]^{p-1}, n \in \mathbf{N}$, then it follows $J_{1}=\sum_{n=1}^{\infty} n^{\frac{q}{r}-1} a_{n}^{q}$. By (12), we have $J<\infty$. If $J=0$, then (15) is obvious value; if $0<J<\infty$, then by (14), we obtain

$$
\begin{aligned}
& \sum_{n=1}^{\infty} n^{\frac{q}{r}-1} a_{n}^{q}=J_{1}=I<c_{r}\left\{\int_{1}^{\infty} x^{\frac{p}{s}-1} f^{p}(x) d x\right\}^{\frac{1}{p}}\left\{\sum_{n=1}^{\infty} n^{\frac{q}{r}-1} a_{n}^{q}\right\}^{\frac{1}{q}}, \\
& J_{1}^{\frac{1}{p}}=\left\{\sum_{n=1}^{\infty} n^{\frac{q}{r}-1} a_{n}^{q}\right\}^{\frac{1}{p}}<c_{r}\left\{\int_{1}^{\infty} x^{\frac{p}{s}-1} f^{p}(x) d x\right\}^{\frac{1}{p}} .
\end{aligned}
$$

Hence we have (15), which is equivalent to (14).

By Hölder's inequality [12], we have

$$
I=\int_{1}^{\infty}\left[x^{\frac{1}{r}-\frac{1}{q}} f(x)\right]\left[x^{-\frac{1}{r}+\frac{1}{q}} \sum_{n=1}^{\infty} \frac{\left|\ln \left(\frac{x}{n}\right)\right| a_{n}}{x+n}\right] d x \leq\left\{\int_{1}^{\infty} x^{\frac{p}{s}-1} f^{p}(x) d x\right\}^{\frac{1}{p}} J_{2}^{\frac{1}{q}} .
$$

By (16), we have (14). On the other hand, suppose that (14) is valid. Setting $f(x):=$ $x^{\frac{q}{r}-1}\left[\sum_{n=1}^{\infty} \frac{\left|\ln \left(\frac{x}{n}\right)\right| a_{n}}{x+n}\right]^{q-1}, x \in[1, \infty)$, then it follows $J_{2}=\int_{1}^{\infty} x^{\frac{p}{s}-1} f^{p}(x) d x$. By (13), we have $J_{2}<\infty$. If $J_{2}=0$, then (16) is obvious value; if $0<J_{2}<\infty$, then by (14), we obtain

$$
\begin{aligned}
& \int_{1}^{\infty} x^{\frac{p}{s}-1} f^{p}(x) d x=J_{2}=I<c_{r}\left\{\int_{1}^{\infty} x^{\frac{p}{s}-1} f^{p}(x) d x\right\}^{\frac{1}{p}}\left\{\sum_{n=1}^{\infty} n^{\frac{q}{r}-1} a_{n}^{q}\right\}^{\frac{1}{q}}, \\
& J_{2}^{\frac{1}{q}}=\left\{\int_{1}^{\infty} x^{\frac{p}{s}-1} f^{p}(x) d x\right\}^{\frac{1}{q}}<c_{r}\left\{\sum_{n=1}^{\infty} n^{\frac{q}{r}-1} a_{n}^{q}\right\}^{\frac{1}{q}} .
\end{aligned}
$$

Hence we have (16), which is equivalent to (14). Therefore (14), (15) and (16) are equivalent.

If the constant factor $c_{r}$ in (14) is not best possible, then there exists a positive number $K$, with $0<K<c_{r}$, such that (14) is still valid if we replace $c_{r}$ by $K$. For $0<\varepsilon<\varepsilon_{0}$, setting $\bar{f}(x)=n^{\frac{1}{r}-\frac{\varepsilon}{p}-1}, \bar{a}_{n}=n^{\frac{1}{s}-\frac{\varepsilon}{q}-1}(n \in N)$, we have

$$
\begin{aligned}
\bar{I} & =\sum_{n=1}^{\infty} \bar{a}_{n} \int_{1}^{\infty} \frac{\left|\ln \left(\frac{x}{n}\right)\right| \bar{f}(x)}{x+n} d x<K\left\{\int_{1}^{\infty} x^{\frac{p}{s}-1} \bar{f}^{p}(x) d x\right\}^{\frac{1}{p}}\left\{\sum_{n=1}^{\infty} n^{\frac{q}{r}-1} \bar{a}_{n}^{q}\right\}^{\frac{1}{q}} \\
& =K\left(\int_{1}^{\infty} x^{-1-\varepsilon} d x\right)^{\frac{1}{p}}\left(1+\sum_{n=2}^{\infty} n^{-1-\varepsilon}\right)^{\frac{1}{q}} \\
& <K\left(\frac{1}{\varepsilon}\right)^{\frac{1}{p}}\left(1+\int_{1}^{\infty} x^{-1-\varepsilon} d x\right)^{\frac{1}{q}}=\frac{K}{\varepsilon}(\varepsilon+1)^{\frac{1}{q}} .
\end{aligned}
$$

By (11) and (21), we have $c_{R}-\varepsilon O(1)<K(\varepsilon+1)^{\frac{1}{q}}$ and for $\varepsilon \rightarrow 0^{+}$, by Fatou lemma [13], we have $c_{r} \leq \lim _{\varepsilon \rightarrow 0^{+}}\left(c_{R}-\varepsilon O(1)\right) \leq K$. This is a contradiction. Hence we can conclude that 
the constant $c_{r}$ in (14) is the best possible. If the constant factors in (15) and (16) are not the best possible, then we can imply a contradiction that the constant factor in (14) is not the best possible by (17) and (19). The theorem is proved.

Remark For $p=q=r=s=2$, (14) reduces to (4). Inequality (4) is a new basic half-discrete Hilbert-type inequality with the non-monotone kernel.

\section{Competing interests}

The authors declare that they have no competing interests.

\section{Authors' contributions}

DX carried out the study, and wrote the manuscript. BY participated in the design of the study, and reformed the manuscript. All authors read and approved the final manuscript.

\section{Acknowledgements}

This work was supported by the Emphases Natural Science Foundation of Guangdong Institutions of Higher Learning College and University (No. 05Z026) and the Natural Science Foundation of Guangdong (7004344).

Received: 19 December 2011 Accepted: 9 August 2012 Published: 30 August 2012

\section{References}

1. Hardy, GH, Littlewood, JE, Polya, G: Inequalities. Cambridge University Press, Cambridge (1952)

2. Xin, D, Yang, B: A basic Hilbert-type inequality. J. Math. (Wuhan) 30(3), 554-560 (2010)

3. Yang, B: On a basic Hilbert-type integral inequality and extensions. Coll. Math. J. 24(1), 87-92 (2008)

4. Yang, B: A mixed Hilbert-type inequality with a best constant factor. Int. J. Pure Appl. Math. 20(3), 319-328 (2005)

5. Yang, B: On a half-discrete Hilbert-type inequality. J. Shandou Univ., Nat. Sci. 26(4), 5-10 (2011)

6. Yang, B: A half-discrete Hilbert-type inequality with a homogeneous kernel of positive degree. J. Zhanjiang Norm. Coll. 32(3), 5-9 (2011)

7. Yang, B: A half-discrete reverse Hilbert-type inequality with non-homogeneous kernel. J. Hunan Inst. Sci. Technol., Nat. Sci. 24(3), 1-4 (2011)

8. Yang, B: On a half-discrete reverse Hilbert-type inequality with a non-homogeneous kernel. J. Inn. Mong. Norm. Univ, Nat. Sci. 40(5), 433-436 (2011)

9. Zhong. W: A mixed Hilbert-type inequality and its equivalent forms. J. Guangdong Univ. Educ. 31(5), 18-22 (2011)

10. Yang, B: A half-discrete Hilbert-type inequality with the non-monotone kernel. J. Jilin Univ., Sci. 49(1), 87-92 (2012)

11. Yang, B: The Norm of Operator and Hilbert-Type Inequalities. Science Press, Beijing (2009)

12. Kuang, J: Applied Inequalities. Shangdong Science Press, Jinan (2004)

13. Kuang, J: Introduction to Real Analysis. Hunan Education Press, Changsha (1996)

doi:10.1186/1029-242X-2012-184

Cite this article as: Xin and Yang: A half-discrete Hilbert-type inequality with the non-monotone kernel and the best constant factor. Journal of Inequalities and Applications 2012 2012:184.

\section{Submit your manuscript to a SpringerOpen ${ }^{\circ}$ journal and benefit from:}

- Convenient online submission

Rigorous peer review

- Immediate publication on acceptance

Open access: articles freely available online

- High visibility within the field

- Retaining the copyright to your article 